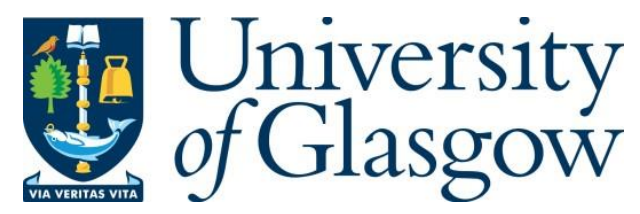

Ma, J., Liu, W. and Zhang, L. (2019) Iterative Transceiver Beamforming of Distributed Relay Networks in Cognitive Radio Networks. In: 2019 IEEE 30th Annual International Symposium on Personal, Indoor and Mobile Radio Communications (PIMRC), Istanbul, Turkey, 08-11 Sep 2019, ISBN 9781538681107.

There may be differences between this version and the published version. You are advised to consult the publisher's version if you wish to cite from it.

http://eprints.gla.ac.uk/188516/

Deposited on: 8 July 2019

Enlighten - Research publications by members of the University of Glasgow http://eprints.gla.ac.uk 


\title{
Iterative Transceiver Beamforming of Distributed Relay Networks in Cognitive Radio Networks
}

\author{
Jingxiao Ma*, Wei Liu*, and Lei Zhang ${ }^{\dagger}$ \\ ${ }^{*}$ Communications Research Group, Department of Electronic and Electrical Engineering, University of Sheffield, UK \\ $\dagger$ Communication Sensing and Imaging Research Group, School of Engineering, University of Glasgow, UK \\ Email: \{jma15,w.liu\}@ sheffield.ac.uk, Lei.Zhang@glasgow.ac.uk
}

\begin{abstract}
This paper investigated the transceiver beamforming problem of multi-pair distributed relay network in a cognitive radio (CR) framework. The goal is to support reliable multi-pair transmissions between secondary users ( $\mathrm{SU}$ ) with assistance of a number of relays, while keeping the total leakage interference on the primary user (PU) under a predefined threshold level. Certain iterative algorithm is proposed to decompose the very difficult original problem into three solvable sub-problems, and after certain number of iteration steps, the obtained transceiver beamformers and relay beamformers can form a group of globally sub-optimal solutions that leads to significant performance enhancement of the secondary network while restricting the leakage interference on PU receiver. Two transmission schemes are considered where the first one only constrain the leakage signal power introduced to $P U$ received, and the second one adds a constraint of the total relay output power. At last, we analyzed the condition, under which the later transmission scheme could be simplified with less computational complexity to solve it.
\end{abstract}

\section{INTRODUCTION}

In a typical cognitive radio (CR) network, one or several secondary users (SUs) are allowed to opportunistically access the spectrum resources licensed to the primary users (PUs) under limitations such as interference perceived at PUs being regulated below a predetermined level. The dynamic access strategy of SUs can work as a promising approach to effectively address the spectrum scarcity and inefficiency of the communication networks. There are mainly two approaches for SUs to access the spectrum resources of PUs, namely spectrum overlay and spectrum underlay. In the former approach, SUs identify and exploit the spectrum holes. Spectrum holes are frequency bands that are allocated to PUs, but during some time intervals, are not utilized by them and thus could be accessed by SUs [1]. In the later approach, the PUs transmit all the time, and the transmission of the SUs are constrained so that their leakage interference powers at the PUs are kept below some predefined allowed threshold [2? ].

In CR networks, distributed beamforming strategy [3, 4] can be used to improve the performance as a spatial processing technique that forms a radiation pattern to well satisfy the requirement of the wireless system. As demonstrated in [5, 6], when single set of SUs is considered, with properly designed beamforming strategy applied in CR networks, both PU and SU sources can have simultaneous communications to their destinations in the same channel. However, when multiple SUs instead of one are accessing the same spectrum resources of the PUs [7? -9], it becomes more challenging to cooperate all the SUs.

In [7], a CR network whose secondary network consists multiple multi-antennal SUs was considered, the authors proposed a simple iterative techniques to solve a convex optimization problem and obtain the optimal beamforming weights for the SUs. However, the process requires hundreds of iteration steps to have a satisfactory performance for the secondary network. [8] studied a similar network where the SU receivers have single antenna and the authors proposed another iterative algorithm that can converge after a much lower number of iteration steps. Following that, the same network as in [8] was considered in [9], with the partial CSI error being taken into consideration. Moreover, massive MIMO reciprocity-based precoder in a CR approach was proposed in [?] to increase the performance and reliability of the secondary network.

In this paper, unlike the former research in literature, we consider multi-pair communications between SUs in the secondary networks, where several multi-antenna user pairs access the spectrum resources of the PUs for their own twoway communications. Furthermore, multiple single-antenna idle devices within the same region of PUs are utilized as distributed relay nodes and assist the SU transmissions with simple amplify and forward protocol. By using this arrangement, proper iterative transceiver beamforming design, which has demonstrated to be strongly capable to increase the SINR of each user pair in a relay beamforming network [10], could be utilized on the SUs and relay nodes to adaptively increase quality of service $(\mathrm{QoS})$ of the secondary network while keeping the leakage interference at PU receiver under a predefined level. In our considered two-stage communication network, we assume that the PUs are having one-way communication and accordingly only the PU receiver are interfered by the communication of SUs. We also assume the transmissions from SU user nodes to SU relay nodes do not cause impermissible interference at the PUs; therefore, in our considered scenario, the leakage signal at PU receiver is only considered in the second transmission stage of SUs when SU relay nodes are broadcasting signals back to SU users. The assumption is well satisfied when the PU users are physically far from the SU user pairs but close from the SU relay nodes.

This paper will be organized as follows. In Section II, the system model is introduced. Then, the iterative transceiver beamforming algorithm is derived in Section III. Following that, simulation results are demonstrated in Section IV. Finally, Section V concludes this paper.

\section{System MODEL}

As shown in Fig II, we consider a time-slotted dualhop distributed $\mathrm{CR}$ relay network with multipair two-way 
communications between $K$ multiple-antenna SU nodes $\left(X_{a}\right.$, $X_{b}$ ), where multiple single-antenna CR relay nodes (R) help forward the information stream, and we also assume that the direct link between source and destination nodes does not exist. The transmission is divided into two time-slotted stages. In the first stage, the SUs broadcast their information streams to all $\mathrm{CR}$ relay nodes with transmit beamforming and their weights are denoted by $\mathbf{a}_{i}$ and $\mathbf{b}_{i}\left(\in \mathbb{C}^{N \times 1}, i=1, \ldots, K\right)$, and in the second stage, each CR relay node forward the received signal back to all the SUs with relay beamforming, which assures that no impermissible interference be caused at the primary destination. Following that, the received signal undergos receive beamforming, denoted by $\mathbf{c}_{i}$ and $\mathbf{d}_{i}\left(\in \mathbb{C}^{N \times 1}\right.$, $i=1, \ldots, K)$, at $X_{a, i}$ and $X_{b, i}$ sides, respectively.

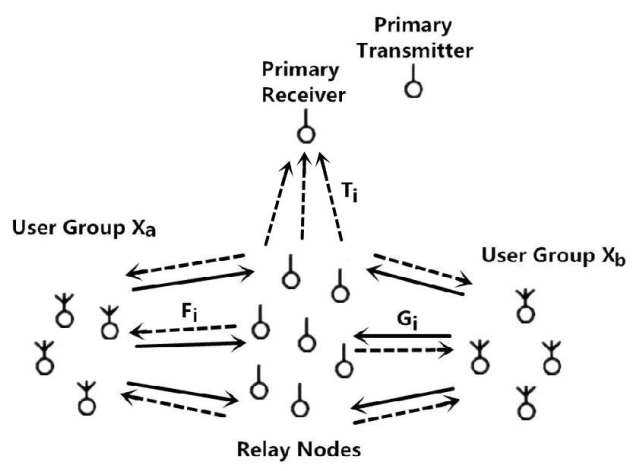

Fig. 1. The considered time-slotted dual-hop multipair two-way distributed relay network.

We denote the SU-source-to-relay channel from $X_{a, i}$ and $X_{b, i}$ to the relay nodes by $\mathbf{F}_{i}, \mathbf{G}_{i} \in \mathbb{C}^{M \times N}$, respectively. We further assume the transmission channels are reciprocal and quasi-stationary, so that the channel gains remain unchanged during the two time slot phases. And the received signal at the relay nodes can be represented by $\mathbf{r} \in \mathbb{C}^{M \times 1}$,

$$
\mathbf{r}=\sum_{i=1}^{K} \mathbf{F}_{i} \mathbf{a}_{i} x_{a, i}+\sum_{i=1}^{K} \mathbf{G}_{i} \mathbf{b}_{i} x_{b, i}+\mathbf{n}_{R},
$$

where the complex Gaussian noise vector of relay nodes are represented by $\mathbf{n}_{R} \in \mathbb{C}^{M \times 1}$ with the distribution $\mathcal{C} \mathcal{N}\left(\mathbf{0}, \sigma_{r}^{2} \mathbf{I}\right)$. Then, each relay node amplifies the received signal to generate the transmit signal $\mathbf{r}_{T}$ as

$$
\mathbf{r}_{T}=\mathbf{W r},
$$

where the relay weights matrix $\mathbf{W} \in \mathbb{C}^{M \times M}$ is diagonal and we use an $M \times 1$ vector $\mathbf{w}=\left[w_{1} w_{2} \ldots w_{M}\right]^{H}$ to denote its diagonal entries. Next, in the second time slot, the relay nodes broadcast $\mathbf{r}_{T}$ to all the SUs. We use $y_{a, i}$ and $y_{b, i}$ to represent the signal received by $X_{a, i}$ and $X_{b}, i$, respectively, with

$$
\begin{aligned}
y_{a, i}=\underbrace{\mathbf{c}_{i} \mathbf{F}_{i}^{T} \mathbf{W} \mathbf{G}_{i} \mathbf{b}_{i} x_{b, i}}_{\text {Desired signal }}+\underbrace{\mathbf{c}_{i} \mathbf{F}_{i}^{T} \mathbf{W} \mathbf{F}_{i} \mathbf{a}_{i} x_{a, i}}_{\text {Self Interference }}+\mathbf{c}_{i} \mathbf{F}_{i}^{T} \mathbf{W} \mathbf{n}_{R} \\
+\mathbf{c}_{i} \mathbf{n}_{a, i}+\underbrace{\mathbf{c}_{i} \mathbf{W} \mathbf{F}_{i}^{T} \sum_{j \neq i}^{K}\left(\mathbf{F}_{j} \mathbf{a}_{j} x_{a, j}+\mathbf{G}_{j} \mathbf{b}_{j} x_{b, j}\right)}_{\text {IPI }},
\end{aligned}
$$

$y_{b, i}$ can be similarly expressed, where $\mathbf{n}_{a, i} \in \mathbb{C}^{N \times 1}$ is the additive white complex Gaussian noise vector at the user node, with the distribution $\mathcal{C N}\left(\mathbf{0}, \sigma_{u}^{2} \mathbf{I}\right)$. The receive beamforming vector $\mathbf{c}_{i}$ is assumed to be unit vector $\left(\left\|\mathbf{c}_{i}\right\|^{2}=1\right)$.

Since its own transmitted signal is known by each user node, the self interference (SI) in (3) can be removed through some standard adaptive filtering techniques [11]. For simplicity, they are ignored in the following derivation.

Then, we use $y^{(P U)}$ to denote the leakage signal introduced by $\mathrm{CR}$ relays at the primary receiver,

$$
\begin{aligned}
y^{(P U)} & =\mathbf{t}_{P} \mathbf{W r} \\
& =\mathbf{t}_{P} \sum_{i=1}^{K} \mathbf{W} \mathbf{F}_{i} \mathbf{a}_{i} x_{a, i}+\mathbf{t}_{P} \sum_{i=1}^{K} \mathbf{W G}_{i} \mathbf{b}_{i} x_{b, i}+\mathbf{t}_{P} \mathbf{n}_{R}
\end{aligned}
$$

where $\mathbf{t}_{P} \in \mathbb{C}^{M \times 1}$ represents the relay-to-PU channel (interference channel).

\section{ITERATIVE BEAMFORMING ALGORITHM FOR COGNITIVE NeTwORKS}

In the following, two transceiver beamforming schemes will be considered for this multipair two-way cognitive network with distributed relays. In our first scheme, the aim is to maximize the SINR at each SU node, while ensuring the leakage signal introduced by CR relays at the primary receiver does not exceed a predefined threshold level. In the second one, a total relay output power constraint is added.

Taking user $X_{a},{ }_{i}$ as an example. From (3), SINR at this user can be expressed as follows,

$$
\operatorname{SIN} R_{a, i}=\frac{\mathbf{c}_{i}^{H} \mathbf{F}_{i}^{T} \mathbf{Q}_{a, i}^{(S)} \mathbf{F}_{i}^{*} \mathbf{c}_{i}}{\sigma_{u}^{2}+\mathbf{c}_{i}^{H} \mathbf{F}_{i}^{T} \mathbf{Q}_{a, i}^{(N)} \mathbf{F}_{i}^{*} \mathbf{c}_{i}+\underbrace{\mathbf{c}_{i}^{H} \mathbf{F}_{i}^{T} \mathbf{Q}_{a, i}^{(I)} \mathbf{F}_{i}^{*} \mathbf{c}_{i}}_{\mathrm{IPI}}},
$$

where,

$$
\begin{aligned}
& \mathbf{Q}_{a, i}^{(I)}=P s \cdot \sum_{j \neq i}^{K}\left(\mathbf{W} \mathbf{F}_{j} \mathbf{a}_{j} \mathbf{a}_{j}^{H} \mathbf{F}_{j}^{H} \mathbf{W}^{H}+\mathbf{G}_{j} \mathbf{b}_{j} \mathbf{b}_{j}^{H} \mathbf{G}_{j}^{H} \mathbf{W}^{H}\right), \\
& \mathbf{Q}_{a, i}^{(N)}=\sigma_{r}^{2} \cdot \mathbf{W} \mathbf{W}^{H}, \mathbf{Q}_{a, i}^{(S)}=P s \cdot \mathbf{W} \mathbf{G}_{i} \mathbf{b}_{i} \mathbf{b}_{i}^{H} \mathbf{G}_{i}^{H} \mathbf{W}^{H} .
\end{aligned}
$$

In our design, we propose to maximize the SINR of each user node while suppressing the interference that is introduced to the primary user node, whether under a sum relay output power constraint or not. Therefore, we can write the overall system formulation for the $i$ th user as follows,

$$
\begin{aligned}
& \max _{\substack{\mathbf{a}_{k}, \mathbf{b}_{k}, \mathbf{c}_{i}, \mathbf{W} \\
(k=1, \ldots, K)}} S I N R_{a, i}, \\
& \text { s.t. }\left\|\mathbf{c}_{i}\right\|^{2}=1,\left\|\mathbf{a}_{k}\right\|^{2} \leq P_{S},\left\|\mathbf{b}_{k}\right\|^{2} \leq P_{S}, \\
&\left(P_{\text {relay }} \leq P_{r}\right), \mathbb{E}\left[y^{(P U)}\right] \leq P_{\text {leak }}
\end{aligned}
$$

where $P_{\text {relay }}$ represents the sum relay output power, and the inequality $P_{\text {relay }} \leq P_{r}$ is presented only in our second scheme in this paper.

As we can see from (7), when all the user are considered altogether, the overall problem will become extremely difficult, if not impossible, to solve. Therefore, as an alternative 
we decide not to jointly solve problem (7) together with other $2 K-1$ similar problems, but to decompose the problem into three sub-problems, each of which is carefully designed based on the role of the transceiver beamforming vectors and the relay coefficients in the SINR expression and in the leakage interference. Note that, although the solution to the three subproblems will very unlikely be the actual global solution of problem, it can provide a rather satisfactory performance. Such a strategy will also help us find a direct solution that can strictly constrain the leakage interference introduced to PU receiver.

\section{A. Iteration Step on the Transmit Part}

In the first iteration steps, we do not consider the contribution of transmit beamformer to the leakage interference according to two reasons. Firstly the first-stage transmission does not cause impermissible interference at PUs, and secondly although the transmit beamforming vectors do affect the leakage interference introduced by the relay nodes at the second transmission stage, proper designs of the transmit beamformers to reduce the leakage interference will result in significant performance degradation at each user node. The reason is that in our transceiver design, the transmit beamforming vectors of one user pair are directly related to the desired signal power of their own transmission. Accordingly, in our design, the leakage interference introduced to the PU receiver is only considered in our second iteration step where the relay beamforming vectors are decided.

In the first iteration step, the receive beamforming vectors $\mathbf{c}_{i}, \mathbf{d}_{i}$ and relay weights $\mathbf{W}$ are fixed to an updated value through previous steps; otherwise, an initial value should be assigned to them. Then, we optimize $\mathbf{a}_{i}$ and $\mathbf{b}_{i}$ based on maximizing the power of the desired signal received at $X_{a}, i$ and $X_{b}, i$, respectively, under a transmit power constraint.

$$
\begin{gathered}
\max _{\mathbf{b}_{i}}\left|\mathbf{c}_{i}^{H} \mathbf{F}_{i}^{T} \mathbf{W} \mathbf{G}_{i} \mathbf{b}_{i}\right|^{2}, \text { s.t. }\left\|\mathbf{b}_{i}\right\|^{2} \leq P_{S}, \\
\max _{\mathbf{a}_{i}}\left|\mathbf{d}_{i}^{H} \mathbf{G}_{i}^{T} \mathbf{W} \mathbf{F}_{i} \mathbf{a}_{i}\right|^{2}, \text { s.t. }\left\|\mathbf{a}_{i}\right\|^{2} \leq P_{S} .
\end{gathered}
$$

These two problems have closed-form solutions, given by

$$
\mathbf{a}_{i}=\lambda_{a, i} \cdot \mathbf{F}_{i}^{H} \mathbf{W}^{H} \mathbf{G}_{i}^{*} \mathbf{d}_{i}, \quad \mathbf{b}_{i}=\lambda_{b, i} \cdot \mathbf{G}_{i}^{H} \mathbf{W}^{H} \mathbf{F}_{i}^{*} \mathbf{c}_{i},
$$

where $\lambda_{a, i}$ and $\lambda_{b, i}$ are the power-control scalars

$$
\lambda_{a, i}=\sqrt{\frac{P_{S}}{\left\|\mathbf{F}_{i}^{H} \mathbf{W}^{H} \mathbf{G}_{i}^{*} \mathbf{d}_{i}\right\|^{2}}}, \quad \lambda_{b, i}=\sqrt{\frac{P_{S}}{\left\|\mathbf{G}_{i}^{H} \mathbf{W}^{H} \mathbf{F}_{i}^{*} \mathbf{c}_{i}\right\|^{2}}} .
$$

B. Iteration Step on the Relays - Maximization of SINR at the Secondary Destination

In the second iteration step of our design, the relay weights are decided based on fixed values (either initialized or updated) of $\mathbf{a}_{i}, \mathbf{b}_{i}, \mathbf{c}_{i}$ and $\mathbf{d}_{i}$. We propose two relay strategies associated with our two considered schemes, where the first one aims at suppressing the leakage interference power at the PU receiver, and the second one adds a total relay output power constraint.

In this subsection, we consider our first relay strategy when the designed relay beamforming coefficients aims at enabling the relay nodes to jointly construct a stream transmission environment that can help the users to obtain a better QoS while suppressing the leakage interference power at the PU receiver.

Constructing a diagonal matrix $\mathbf{T}_{P}$ with its diagonal entries being the elements of $\mathbf{t}_{P}$, together with (1) and (2) we can derive the expression of the leakage interference power,

$$
\mathbb{E}\left[y^{(P U)}\right]=\mathbf{w}^{H} \mathbf{Q}_{\text {leak }} \mathbf{w}
$$

where

$$
\mathbf{Q}_{\text {leak }}=\sum_{i=1}^{K} \mathbf{T}_{P} \mathbf{F}_{i} \mathbf{a}_{i} \mathbf{a}_{i}^{H} \mathbf{F}_{i}^{H} \mathbf{T}_{P}^{H}+\sum_{i=1}^{K} \mathbf{T}_{P} \mathbf{G}_{i} \mathbf{b}_{i} \mathbf{b}_{i}^{H} \mathbf{G}_{i}^{H} \mathbf{T}_{P}^{H}
$$

Using the above results, the following formulation is adopted to find the relay weights that optimizes the sum desired signal power received by all the user nodes.

$$
\begin{aligned}
\underset{\mathbf{w}}{\max _{i=1}} & \sum_{i=}^{K}\left(\left|\mathbf{c}_{i}^{H} \mathbf{F}_{i}^{T} \mathbf{W} \mathbf{G}_{i} \mathbf{b}_{i}\right|^{2}+\left|\mathbf{d}_{i}^{H} \mathbf{G}_{i}^{T} \mathbf{W} \mathbf{F}_{i} \mathbf{a}_{i}\right|^{2}\right), \\
\text { s.t. } & \mathbf{w}^{H} \mathbf{Q}_{\text {leak }} \mathbf{w} \leq P_{\text {leak }} .
\end{aligned}
$$

where $P_{\text {leak }}$ is the pre-defined threshold for the leakage signal power at the primary receiver. Then we can transform this problem into an eigenvalue problem [12] where closed-form solutions can be obtained as,

$$
\mathbf{w}=\lambda \rho\left\{\mathbf{Q}_{\text {leak }}^{-1} \mathbf{Q}_{R}\right\},
$$

where $\rho\{\cdot\}$ denotes the principle eigenvector of a matrix, $\lambda$ is a power control scalar decided by $P_{\text {leak }}$, and $\mathbf{Q}_{R}$ has the following definition.

$$
\mathbf{Q}_{R}=\sum_{i=1}^{K}\left(\mathcal{G}_{i} \mathfrak{f}_{i}^{\prime} \mathfrak{f}_{i}^{\prime H} \mathcal{G}_{i}^{H}+\mathcal{F}_{i} \mathfrak{g}_{i}^{\prime} \mathfrak{g}_{i}^{\prime H} \mathcal{F}_{i}^{H}\right)
$$

where $\mathcal{G}_{i}$ and $\mathcal{F}_{i} \in \mathbb{C}^{M \times M}$ are diagonal matrices with their main diagonal entries corresponding to $\mathbf{G}_{i} \mathbf{b}_{i}$ and $\mathbf{F}_{i} \mathbf{a}_{i}$, respectively, $\mathfrak{g}_{i}^{\prime}=\mathbf{G}_{i} \mathbf{d}_{i}^{*}$ and $\mathfrak{f}_{i}^{\prime}=\mathbf{F}_{i} \mathbf{c}_{i}^{*}$.

We use $\overline{\mathbf{w}}$ to represent the normalized principle eigenvector of $\mathbf{Q}_{\text {leak }}^{\prime-1} \mathbf{Q}_{R}$ and the power control scalar $\lambda$ can be obtained by

$$
\lambda=\sqrt{\frac{P_{\text {leak }}}{\overline{\mathbf{w}}^{H} \mathbf{Q}_{\text {leak }} \overline{\mathbf{w}}}} .
$$

C. Iteration Step on the Relays - Maximization of SINR at the Secondary Destination with Total Relay Output Power Constraint

Now consider the relay beamforming problem with total relay output power constraint in this subsection. Using user $X_{a, i}$ as an example, from (2) and (3), we can write the sum relay power $P_{\text {relay }}$ as,

$$
\begin{aligned}
P_{\text {relay }} & =\mathbf{w}^{H}\left(\sigma_{r}^{2} \mathbf{I}_{M}+\sum_{i=1}^{K} \mathcal{G}_{i} \mathcal{G}_{i}^{H}+\sum_{i=1}^{K} \mathcal{F}_{i} \mathcal{F}_{i}^{H}\right) \mathbf{w} \\
& =\mathbf{w}^{H} \mathbf{Q}_{P} \mathbf{w}
\end{aligned}
$$


where $\mathbf{Q}_{P}$ is a diagonal matrix. The beamforming problem can now be represented as,

$$
\begin{aligned}
\max _{\mathbf{w}} & \sum_{i=1}^{K}\left(\left|\mathbf{c}_{i}^{H} \mathbf{F}_{i}^{T} \mathbf{W} \mathbf{G}_{i} \mathbf{b}_{i}\right|^{2}+\left|\mathbf{d}_{i}^{H} \mathbf{G}_{i}^{T} \mathbf{W} \mathbf{F}_{i} \mathbf{a}_{i}\right|^{2}\right), \\
\text { s.t. } & \mathbf{w}^{H} \mathbf{Q}_{\text {leak }} \mathbf{w} \leq P_{\text {leak }} \\
& \mathbf{w}^{H} \mathbf{Q}_{P} \mathbf{w} \leq P_{r}
\end{aligned}
$$

where $P_{r}$ represents the sum relay power constraint.

Taking user $X_{a}, i$ as an example, we rewrite (5) and (6) with respect to the definition of $\mathcal{G}_{i}, \mathcal{F}_{i}, \mathfrak{g}_{i}^{\prime}$ and $\mathfrak{f}_{i}^{\prime}$.

$$
S I N R_{a, i}=\frac{\mathbf{w}_{i}^{H} \overline{\mathbf{Q}}_{a, i}^{(S)} \mathbf{w}_{i}}{\sigma_{u}^{2}+\mathbf{w}_{i}^{H} \overline{\mathbf{Q}}_{a, i}^{(N)} \mathbf{w}_{i}+\mathbf{w}_{i}^{H} \overline{\mathbf{Q}}_{a, i}^{(I)} \mathbf{w}_{i}},
$$

where,

$$
\begin{aligned}
& \overline{\mathbf{Q}}_{a, i}^{(I)}=P s \cdot \sum_{j \neq i}^{K}\left(\mathcal{F}_{j} \mathfrak{f}_{i}^{\prime} \mathfrak{f}_{i}^{\prime H} \mathcal{F}_{j}^{H}+\mathcal{G}_{j} \mathfrak{f}_{i}^{\prime} \mathfrak{f}_{i}^{\prime} \mathcal{G}_{j}^{H}\right), \\
& \overline{\mathbf{Q}}_{a, i}^{(N)}=\sigma_{r}^{2} \cdot \mathfrak{f}_{i}^{\prime} \mathfrak{f}_{i}^{\prime H}, \overline{\mathbf{Q}}_{a, i}^{(S)}=P s \cdot \mathcal{G}_{i} \mathfrak{f}_{i}^{\prime} \mathfrak{f}_{i}^{\prime H} \mathcal{G}_{i}^{H} .
\end{aligned}
$$

Then, using (22) and (23), and introducing an auxiliary variable $\mu<0$ [13], (21) can be transformed into

$$
\begin{aligned}
\max _{\mathbf{w}, \mu} & \mu \\
\text { s.t. } & \frac{\mathbf{w}_{i}^{H} \overline{\mathbf{Q}}_{a, i}^{(S)} \mathbf{w}_{i}}{\sigma_{u}^{2}+\mathbf{w}_{i}^{H} \overline{\mathbf{Q}}_{a, i}^{(N)} \mathbf{w}_{i}+\mathbf{w}_{i}^{H} \overline{\mathbf{Q}}_{a, i}^{(I)} \mathbf{w}_{i}} \geq \mu^{2} \\
& \mathbf{w}^{H} \mathbf{Q}_{\text {leak }} \mathbf{w} \leq P_{\text {leak }} \\
& \mathbf{w}^{H} \mathbf{Q}_{P} \mathbf{w} \leq P_{r}
\end{aligned}
$$

Denoting $\mathbf{h}=\sqrt{P s} \cdot \mathcal{G}_{i} \mathfrak{f}_{i}^{\prime}$, (21) can be changed into a standard second order cone programming (SOCP) [13] as

$$
\begin{array}{cl}
\max _{\mathbf{w}, \mu} & \mu \\
\text { s.t. } & \mu\|\mathbf{U} \tilde{\mathbf{w}}\| \leq \sqrt{P_{s}} \tilde{\mathbf{w}}^{H} \tilde{\mathbf{h}} \\
& \left\|\tilde{\mathbf{V}}_{Q} \tilde{\mathbf{w}}\right\| \leq P_{N} \\
& \left\|\tilde{\mathbf{V}}_{P} \tilde{\mathbf{w}}\right\| \leq P_{0} \\
& \tilde{\mathbf{w}}_{\text {first }}=1
\end{array}
$$

where $\tilde{\mathbf{w}}=\left[1, \mathbf{w}^{T}\right]^{T}, \quad \tilde{\mathbf{V}}_{P}=\left[\mathbf{0}_{M \times 1}, \mathbf{V}_{P}\right], \quad \tilde{\mathbf{V}}_{Q}=$ $\left[\mathbf{0}_{M \times 1}, \mathbf{V}_{Q}\right], \tilde{\mathbf{h}}=\left[0, \mathbf{h}^{T}\right]^{T}$, and $\tilde{\mathbf{w}}_{\text {first }}$ denotes the first element of $\tilde{\mathbf{w}}$, with

$$
\begin{aligned}
& \tilde{\mathbf{Q}}=\left[\begin{array}{cc}
\sigma_{v}^{2} & \mathbf{0}_{1 \times M} \\
\mathbf{0}_{M \times 1} & \overline{\mathbf{Q}}_{a, i}^{(I)}+\overline{\mathbf{Q}}_{a, i}^{(N)}
\end{array}\right]=\mathbf{U}^{H} \mathbf{U} \\
& \mathbf{Q}_{\text {leak }}=\mathbf{V}_{Q}{ }^{H} \mathbf{V}_{Q} \\
& \mathbf{Q}_{P}=\mathbf{V}_{P}{ }^{H} \mathbf{V}_{P}
\end{aligned}
$$

Note that $\mathbf{U}, \mathbf{V}_{Q}$ and $\mathbf{V}_{P}$ are the Cholesky factorization product of matrix $\tilde{\mathbf{Q}}, \mathbf{D}$ and $\mathbf{Q}_{\text {leak }}$, respectively. The SOCP (22) can be solved by firstly reducing it to a SOCP feasibility problem by assigning a value of $\mu$ using the bisection search procedure [14] and then the interior point method [13] or some other advanced interior-point-based methods can be used to solve it, such as the SeDuMi package [15], which produces a feasibility certificate if the problem is feasible.
Using the bisection search procedure and interior-pointbased methods to solve problem (23) requires several rounds of iteration and thus requires relatively complicated computation resources. However, under particular conditions, the problem can be reduced to one of the two following subschemes, and as our simulation results will demonstrate, with particular settings in our considered network those conditions can be met with high probabilities. The two sub-schemes are given as,

$$
\begin{array}{rl}
\max _{\mathbf{w}} & S I N R \\
\text { s.t. } & \mathbf{w}^{H} \mathbf{Q}_{\text {leak }} \mathbf{w} \leq P_{\text {leak }}
\end{array}
$$

and

$$
\begin{array}{ll}
\max _{\mathbf{w}} & S I N R \\
\text { s.t. } & \mathbf{w}^{H} \mathbf{Q}_{P} \mathbf{w} \leq P_{r}
\end{array}
$$

Both of them can be solved using the same approach as in Section III-B. Denote the solution to problem (24) and (25) as $\mathbf{w}_{\text {opt } 1}$ and $\mathbf{w}_{\text {opt } 2}$, respectively. Under the following conditions, problem (22) can be transformed into either of the above subschemes.

Condition 1: If $\quad \mathbf{w}_{o p t 1}^{H} \mathbf{Q}_{P} \mathbf{w}_{\text {opt } 1} \leq P_{r} \quad$ and $\mathbf{w}_{\text {opt } 2}^{H} \mathbf{Q}_{\text {leak }} \mathbf{w}_{\text {opt2 }}>P_{\text {leak }},(22)$ is transformed to subscheme (24), and the solution is $\mathbf{w}_{\text {opt } 1}$.

Condition 2: If $\mathbf{w}_{o p t 1}^{H} \mathbf{Q}_{P} \mathbf{w}_{\text {opt } 1}>P_{r}$ and $\mathbf{w}_{\text {opt } 2}^{H} \mathbf{Q}_{\text {leak }} \mathbf{w}_{\text {opt2 }} \leq P_{\text {leak }},(22)$ is transformed to subscheme (25), and the solution is $\mathbf{w}_{\text {opt } 2}$.

Condition 3: $\quad \mathbf{w}_{o p t 1}^{H} \mathbf{Q}_{P} \mathbf{w}_{o p t 1} \leq P_{r} \quad$ and $\mathbf{w}_{\text {opt2 }}^{H} \mathbf{Q}_{\text {leak }} \mathbf{w}_{\text {opt2 }} \leq P_{\text {leak }}$ can only be satisfied when $\mathbf{w}_{\text {opt } 1}^{H} \mathbf{Q}_{P} \mathbf{w}_{\text {opt } 1}=P_{r}$, and $\mathbf{w}_{\text {opt } 2}^{H} \mathbf{Q}_{\text {leak }} \mathbf{w}_{\text {opt } 2}=P_{\text {leak }}$. In this case, $\mathbf{w}_{\text {opt } 1}$ and $\mathbf{w}_{\text {opt } 2}$ are identical.

Condition 4: If $\mathbf{w}_{o p t 1}^{H} \mathbf{Q}_{P} \mathbf{w}_{\text {opt } 1}>P_{r}$ and $\mathbf{w}_{\text {opt2 }}^{H} \mathbf{Q}_{\text {leak }} \mathbf{w}_{\text {opt2 }}>P_{\text {leak }}$, (22) cannot be transformed into either of the sub-schemes, and it remains being solved as SOCP.

\section{Iteration Step on the Receive Part}

Now the values of the two transmit beamforming vectors $\mathbf{a}_{i}, \mathbf{b}_{i}$ and the relay coefficients $\mathbf{w}$ are all updated. Next are the two beamforming vectors $\mathbf{c}_{i}$ for user $X_{a, i}$ and $\mathbf{d}_{i}$ for user $X_{b, i}$. Since the receive beamforming vectors will not produce any leakage interference to the PU receiver, they are decided to optimize SINR of each user.

Recalling the SINR expression in (5) and (6), the receive beamforming vector that optimizes SINR of each user can be derived as,

$$
\mathbf{c}_{i}=\rho\left\{\boldsymbol{\Theta}_{a, i}\right\}, \quad \mathbf{d}_{i}=\rho\left\{\boldsymbol{\Theta}_{b, i}\right\},
$$

where

$$
\begin{aligned}
& \boldsymbol{\Theta}_{a, i}=\left(\boldsymbol{\Xi}_{a, i}\right)^{-1} \mathbf{F}_{i}^{T} \mathbf{Q}_{a, i}^{(S)} \mathbf{F}_{i}^{*} \\
& \boldsymbol{\Xi}_{a, i}=\sigma_{u}^{2} \mathbf{I}_{N}+\mathbf{F}_{i}^{T} \mathbf{Q}_{a, i}^{(N)} \mathbf{F}_{i}^{*}+\mathbf{F}_{i}^{T} \mathbf{Q}_{a, i}^{(I)} \mathbf{F}_{i}^{*}
\end{aligned}
$$




\section{E. Iteration Algorithm Summary}

In our proposed algorithms, we have collaboratively maximized the SINR of each SU by the transmit beamformer, receive beamformer and relay nodes together, while the leakage interference signal introduced to the PU receiver is reduced by carefully designed relay beamforming vectors. The three above iteration steps are repeated until reaching the stopping criterion, which is defined by a preset maximum iteration number $\left(n_{t}\right)$ or some convergence requirement (defined by a preset small positive real number $\epsilon$ ).

The iteration steps of the two proposed schemes are summarized in Iteration Algorithm Summary A and Iteration Algorithm Summary B for the two considered schemes as follows.

\section{Iteration Algorithm Summary A}

1) Initialization: $\mathbf{c}_{i}, \mathbf{d}_{i}=\left[\delta_{N} \delta_{N} \cdots \delta_{N}\right] \in \mathbb{C}^{1 \times N}$, where $\delta_{N}=1 / \sqrt{N}, \mathbf{w}=\left[\delta_{M} \delta_{M} \cdots \delta_{M}\right]$, where $\delta_{M}=1 / \sqrt{M}$, and set $\mathrm{t}=1$.

2) Update $\mathbf{a}_{i}$ and $\mathbf{b}_{i}$ based on (9) and (10).

3) Update $w$ based on (14) and (16).

4) Update $\mathbf{c}_{i}$ and $\mathbf{d}_{i}$ based on (26) and (27).

5) If $\left|\mathbf{x}_{i}^{(t)}-\mathbf{x}_{i}^{(t-1)}\right|^{2}<\epsilon$ (considered to be converged) or $t>n_{t}\left(\mathbf{x} \leftarrow \mathbf{c}\right.$ for user $X_{a, i}$ and $\mathbf{x} \leftarrow \mathbf{d}$ for user $\left.X_{b, i}\right)$, iteration stops; otherwise, set $t=t+1$ and go to step 2).

\section{Iteration Algorithm Summary B}

1) Initialization: $\mathbf{c}_{i}, \mathbf{d}_{i}=\left[\delta_{N} \delta_{N} \cdots \delta_{N}\right] \in \mathbb{C}^{1 \times N}$, where $\delta_{N}=1 / \sqrt{N}, \mathbf{w}=\left[\delta_{M} \delta_{M} \cdots \delta_{M}\right]$, where $\delta_{M}=1 / \sqrt{M}$, and set $\mathrm{t}=1$.

2) Update $\mathbf{a}_{i}$ and $\mathbf{b}_{i}$ based on (9) and (10)

3) Obtain $\mathbf{w}_{\text {opt } 1}$ and $\mathbf{w}_{\text {opt } 2}$ by solving 24 and 25 , respectively. Update $\mathbf{w}$ based on which of the four conditions that $\mathbf{w}_{\text {opt } 1}$ and $\mathbf{w}_{\text {opt } 2}$ satisfy.

4) Update $\mathbf{c}_{i}$ and $\mathbf{d}_{i}$ based on (26) and (27).

5) If $\left|\mathbf{x}_{i}^{(t)}-\mathbf{x}_{i}^{(t-1)}\right|^{2}<\epsilon$ (considered to be converged) or $t>n_{t}\left(\mathbf{x} \leftarrow \mathbf{c}\right.$ for user $X_{a, i}$ and $\mathbf{x} \leftarrow \mathbf{d}$ for user $\left.X_{b, i}\right)$, iteration stops; otherwise, set $t=t+1$ and go to step 2).

\section{Simulation Results}

In our simulations, we consider the cognitive network with multi-pair communication between SUs, with the number of user pairs being set as $K=3$. The transmission channels between SUs to relays and relays to PU are quasi-static i.i.d. Rayleigh fading channels; the noise variance at any node is set as $1\left(\sigma_{r}^{2}=\sigma_{u}^{2}=1\right)$ and we set the source power at $0 \mathrm{~dB}\left(P_{S}=1\right.$, compensating the unconsidered large-scale fading). The leakage threshold $P_{\text {leak }}$ is determined by $S N R_{L}=P_{l e a k} / \sigma_{r}^{2}$, while the total relay output power constraint is determined by $S N R_{R}=P_{r} / \sigma_{r}^{2}$. The value of $\epsilon=0.01$ is chosen to determine the convergence of the iterative process, and $n_{t}$ represents the maximum number of iteration rounds. And in our simulations, we consider the very worst situation when the PU receiver is located close to the

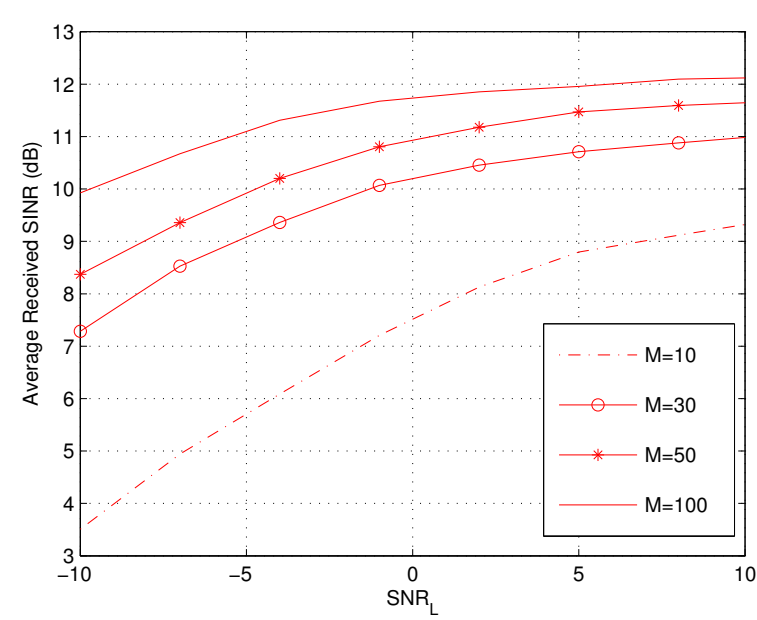

Fig. 2. SINR performance versus the leakage power threshold and number of relays $\left(\mathrm{N}=5, \mathrm{~K}=3, n_{t}=5\right)$, first scheme.

relays, and thus the additional path loss between relays and PU receiver is assumed to be $0 \mathrm{~dB}$.

In Fig. 2, we present the average SINR performance of the first proposed approach, versus the leakage interference power threshold at the primary receiver, for different number of relays. It can be seen that the SINR performance is very satisfactory considering that the PU receiver is located close to the relays. As the threshold $S N R_{L}$ is set higher, the performance gets better; however, when a large number of relays are included in the network $(M=100)$, the SINR difference at $S N R_{L}=-10 \mathrm{~dB}$ and $S N R_{L}=10 \mathrm{~dB}$ is only $2 \mathrm{~dB}$, which indicates that increasing the number of relays can dramatically reduce the interference introduced to the PU receiver, when total relay output power is not restricted. The reason is that, as indicated in [10], in such iterative transceiver algorithms, when the number of relays increases, the total relay output power required to achieve the same SINR can be greatly reduced and so is the total interference introduced to the PU receiver.

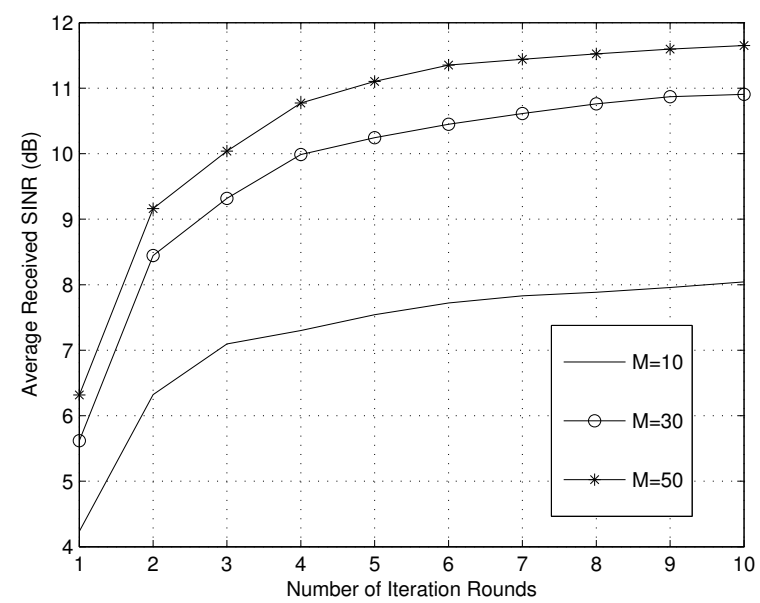

Fig. 3. SINR performance versus the number of iteration rounds $\left(P_{\text {leak }}=0\right.$ $\mathrm{dB}, \mathrm{N}=5, \mathrm{~K}=3$ ), first scheme. 
Fig. 3 demonstrates the SINR performance of the first proposed approach in terms of number of iteration rounds. As can be seen, the SINR performance could be significantly improved within the first few iteration rounds. It shows that our proposed iterative transceiver beamforming algorithm can effectively coordinate the SUs and relay transmissions and improve their transmission QoS without requiring a large number of iteration steps.

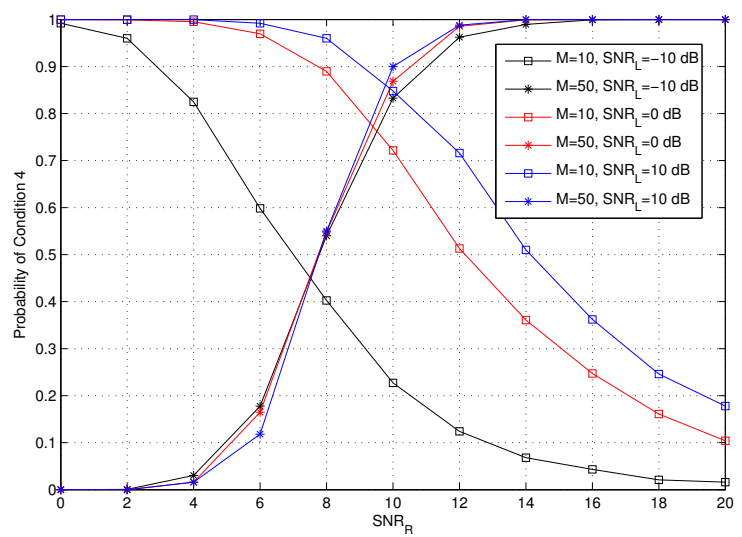

Fig. 4. Probability of Condition 4 , second scheme $(\mathrm{N}=5, \mathrm{~K}=3)$.

In Fig. 4, the probability of our second scheme satisfying Condition 4 is depicted, in which the relay beamforming vectors can only be obtained by solving an SOCP with higher computational complexity. As shown in the figure, the probability peak is always 1 where the original scheme can not be transformed at all, and the peak is shifted as the network settings change. Carefully choosing the network settings to avoid the transmission network from having high probability of Condition 4 can help reduce the computational complexity in our scenario. As can be noticed from the figure, to achieve this, one way is to utilize more relay nodes in the secondary network while decreasing the relay output power budget, and the other way is to have high relay output power budget while decreasing the relay number. Not hard to find, both of the two way will lead to SINR performance degradation. Accordingly, in our proposed method, trade-off must be made and the simulation result can be used as a guidance.

\section{Summary}

In this paper, transceiver beamforming problem in a cognitive network with multi-pair communication between SUs is studied. With our proposed iterative algorithm, the very difficult beamforming problem can be decomposed into three sub-problem, each of which leads to a value update of the beamforming vectors. Through iteration, an overall suboptimal solution can be obtained to increase the SINR performance of each SU while the leakage interference introduced to PUs is strictly restricted. When a total relay output power constraint is considered, the transceiver beamforming problem can be either solved as an SOCP or transformed into two simpler sub-schemes under some specific conditions. The probability of performing such a transformation is investigated and simulation results are provided to guide the network settings.

\section{REFERENCES}

[1] A. Mesodiakaki, F. Adelantado, L. Alonso, and C. Verikoukis, "Performance analysis of a cognitive radio contention-aware channel selection algorithm," IEEE Transactions on Vehicular Technology, vol. 64, no. 5, pp. 1958-1972, 2015.

[2] — "Energy-efficient user association in cognitive heterogeneous networks," IEEE Communications Magazine, vol. 52, no. 7, pp. 22-29, 2014.

[3] L. Zhang, W. Liu, A. u. Quddus, M. Dianati, and R. Tafazolli, "Adaptive distributed beamforming for relay networks based on local channel state information," IEEE Transactions on Signal and Information Processing over Networks, vol. 1, no. 2, pp. 117-128, June 2015.

[4] L. Zhang, W. Liu, and J. Li, "Low-complexity distributed beamforming for relay networks with real-valued implementation," IEEE Transactions on Signal Processing, vol. 61, no. 20, pp. 5039-5048, 2013.

[5] L. Zhang, Y.-C. Liang, and Y. Xin, "Joint beamforming and power allocation for multiple access channels in cognitive radio networks," IEEE Journal on Selected Areas in Communications, vol. 26, no. 1, 2008.

[6] L. Zhang, Y.-C. Liang, Y. Xin, and H. V. Poor, "Robust cognitive beamforming with partial channel state information," IEEE Transactions on Wireless Communications, vol. 8, no. 8, 2009.

[7] M. Pesavento, D. Ciochina, and A. B. Gershman, "Iterative dual downlink beamforming for cognitive radio networks," in Cognitive Radio Oriented Wireless Networks \& Communications (CROWNCOM), 2010 Proceedings of the Fifth International Conference on. IEEE, 2010, pp. 1-5.

[8] I. Wajid, H. Nikolaeva, and M. Pesavento, "Iterative robust downlink beamforming in cognitive radio networks," in Cognitive Radio Oriented Wireless Networks and Communications (CROWNCOM), 2011 Sixth International ICST Conference on. IEEE, 2011, pp. 375-379.

[9] J.-H. Noh and S.-J. Oh, "Beamforming in a multi-user cognitive radio system with partial channel state information," IEEE Transactions on wireless communications, vol. 12, no. 2, pp. 616-625, 2013.

[10] J. Ma and W. Liu, "Robust iterative transceiver beamforming for multipair two-way distributed relay networks," IEEE Access, vol. 5, pp. 24 656-24 667, 2017.

[11] S. S. Haykin, Adaptive filter theory. Pearson Education India, 2008.

[12] G. H. Golub and C. F. Van Loan, Matrix computations, 3rd ed. Baltimore, MD, USA: Johns Hopkins Univ. Press, 1996.

[13] S. Boyd and L. Vandenberghe, Convex optimization. Cambridge, U.K.: Cambridge Univ. Press, 2004.

[14] V. Havary-Nassab, S. Shahbazpanahi, A. Grami, and Z.Q. Luo, "Distributed beamforming for relay networks based on second-order statistics of the channel state information," IEEE Transactions on Signal Processing, vol. 56, no. 9, pp. 4306-4316, 2008.

[15] J. F. Sturm, "Using sedumi 1.02, a matlab toolbox for optimization over symmetric cones," Optimization methods and software, vol. 11, no. 1-4, pp. 625-653, 1999. 